\title{
Acute effects of different types of oil consumption on endothelial function, oxidative stress status and vascular inflammation in healthy volunteers
}

\author{
Dimitris Tousoulis* $\uparrow$, Nikolaos Papageorgiou $\dagger$, Charalambos Antoniades, Anastasios Giolis, George Bouras, \\ Panagiota Gounari, Elli Stefanadi, Antigoni Miliou, Theodora Psaltopoulou and Christodoulos Stefanadis \\ 1st Cardiology Unit, Hippokration Hospital, Athens University Medical School, Athens, Greece
}

(Received 19 February 2009 - Revised 15 June 2009 - Accepted 25 June 2009 - First published online 25 August 2009)

Consumption of different types of oil may have different effects on cardiovascular risk. The exact role of maize oil, cod liver oil, soya oil and extra virgin olive oil on endothelial function, oxidative stress and inflammation is unknown. We evaluated the effect of acute consumption of these types of oil on endothelial function, oxidative stress and inflammation in healthy adults. Thirty-seven healthy volunteers were randomised to receive an oral amount of each type of oil or water. Endothelial function was evaluated by gauge-strain plethysmography at baseline and 1,2 and $3 \mathrm{~h}$ after consumption. Oxidative stress status was determined by total lipid peroxides (PEROX), while inflammatory process was estimated by measuring the soluble form of vascular adhesion molecule 1. Serum levels of the two previous markers were measured at baseline and $3 \mathrm{~h}$ after oil consumption. Reactive hyperaemia $(\mathrm{RH})$ was significantly decreased after maize oil consumption compared with controls $(P<0 \cdot 05)$. However, the consumption of cod liver oil and soya oil induced a significant improvement of RH after $1 \mathrm{~h}$, compared with controls $(P<0 \cdot 05)$. There was no significant effect of any type of oil consumption on endothelium-independent dilatation, total lipid PEROX and vascular adhesion molecule 1 serum levels. Consumption of maize oil leads to impaired endothelial function, while soya oil and cod liver oil slightly improve endothelial function. However, all types of oils did not affect inflammatory process and systemic oxidative stress, suggesting that their effect on endothelial function may not be mediated by free radicals bioavailability.

Oils: Inflammation: Oxidative stress: Endothelial function

Atherosclerosis is the major source of morbidity and mortality in the developed world, representing the result of several underlying mechanisms and closely related to endothelial function and oxidative stress ${ }^{(1,2)}$. It is well known that the vascular endothelium regulates the balance between vasodilatation and vasoconstriction, including a number of different processes. Whenever this balance is upset, endothelial dysfunction occurs ${ }^{(1,3)}$. In addition, endothelial dysfunction is considered to be an early marker for atherosclerosis. Studies have shown that endothelial dysfunction is present even in the preclinical stage of atherosclerosis ${ }^{(4-6)}$.

Evidence suggests that increased production of reactive oxygen species and vascular inflammation play an important role in endothelial dysfunction. Several vascular sources of superoxide have been implicated including NADPH, xanthine oxidase, lipoxygenases and myeloperoxidase ${ }^{(7,8)}$. Superoxide reacts with $\mathrm{NO}$, resulting in the formation of peroxynitrite ${ }^{(9)}$. Moreover, vascular inflammation plays an important role in the process of endothelial dysfunction mainly though the proinflammatory cytokines, selectins and adhesion molecules ${ }^{(10)}$.

Epidemiological and clinical studies showed that the Mediterranean diet is associated with significantly lower cardiovascular risk. Although it is difficult to distinguish specific dietary factors, data suggest that olive oil, used as primary component of the Mediterranean diet, may play a key role in cardiovascular protection ${ }^{(11)}$. It is still not clear whether other types of oil could be beneficial. Therefore, in the present study, we examined the effects of maize oil, cod liver oil, soya oil and extra virgin olive oil on endothelial function, vascular cell adhesion molecule 1 (VCAM-1) and oxidative stress status in healthy subjects.

\section{Experimental methods}

\section{Study population and protocol}

A total number of thirty-seven healthy young subjects were recruited for the present parallel study. Exclusion criteria were the presence of clinical evidence for atherosclerotic peripheral vascular disease or coronary atherosclerosis, the presence of any acute or chronic inflammatory diseases (e.g. liver diseases) or any other cardiac disease (including coronary artery disease documented by medical history). Subjects with any classic risk factor for atherosclerosis such as smoking, as well as those receiving any systemic medication, were also excluded from the study.

\footnotetext{
Abbreviations: FBF, forearm blood flow; NTG, nitroglycerine; PEROX, peroxides; RH, reactive hyperaemia; VCAM-1, vascular cell adhesion molecule 1.

* Corresponding author: Dr Dimitris Tousoulis, fax + 302107784590 , email drtousoulis@ hotmail.com

$\dagger$ Equally contributed in the present study.
} 
All the participants were randomly allocated into five groups to receive four different types of oil or water, in order to receive an equivalent amount of $50 \mathrm{ml}$ as reported previously $^{(12)}$. Each type of oil had a known macronutrients composition commercially available. Thus, the composition per $100 \mathrm{ml}$ of each type of oil was: extra virgin olive oil $(824 \mathrm{kcal}$ or $3449.9 \mathrm{~kJ}, 91.6 \mathrm{~g}$ fatty acids, $12.8 \mathrm{~g}$ saturated, $70.5 \mathrm{~g}$ monounsaturated and $8.3 \mathrm{~g}$ polyunsaturated); maize oil ( $825 \mathrm{kcal}$ or $3454.1 \mathrm{~kJ}, 92 \mathrm{~g}$ fatty acids, $12 \mathrm{~g}$ saturated, $25 \mathrm{~g}$ monounsaturated and $55 \mathrm{~g}$ polyunsaturated); soya oil (825 kcal or $3454.1 \mathrm{~kJ}, 92 \mathrm{~g}$ fatty acids, $14 \mathrm{~g}$ saturated, $23 \mathrm{~g}$ monounsaturated and $55 \mathrm{~g}$ polyunsaturated); cod liver oil $(830 \mathrm{kcal}$ or $3475.0 \mathrm{~kJ}, 92 \mathrm{~g}$ cod oil, $0.00828 \mathrm{~g}$ EPA, $0.00736 \mathrm{~g}$ DHA, $0.0112 \mathrm{~g}$ vitamin A, $0.0001 \mathrm{~g}$ vitamin D and $0.068 \mathrm{~g}$ vitamin $\mathrm{E}$ ). It is worth to mention that extra virgin olive oil was administered, as minor components of virgin olive oil are thought to be responsible for much of its beneficial effects $^{(13,14)}$. Oils were administered directly from their commercial form (liquid) and not as part of a meal. Endothelial function was evaluated by gauge-strain plethysmography at baseline, 1,2 and $3 \mathrm{~h}$ after oil consumption, and blood samples were obtained at baseline and at $3 \mathrm{~h}$. The group of subjects, who received water, allowed us to correct for any circadian variation of serum sVCAM-1, total lipid peroxides (PEROX) and endothelial function. The protocol was approved by the ethics committee of our institution and a written informed consent was given by each subject.

\section{Forearm blood flow measurements}

In the present study, we evaluated post-ischaemic hyperaemia as an index of endothelial function in the right forearm ${ }^{(15)}$. Briefly, all measurements were performed in the morning in a dark quiet room under controlled temperature $\left(22-24^{\circ} \mathrm{C}\right)$. All subjects abstained from food, tobacco, alcohol and caffeine-containing drinks for at least $12 \mathrm{~h}$ before vascular study. Before measurements were started, subjects were rested in a supine position for $30 \mathrm{~min}$. Forearm blood flow (FBF) was measured using venous occlusion, gauge-strain plethysmography (EC-400, D.E. Hokanson, Inc., Bellevue, WA, USA) as previously described $^{(15)}$. Briefly, a mercury-in-silastic strain gauge, which had been electrically calibrated, was positioned on the widest part of the forearm. The pressure of the collecting cuff was set at $40 \mathrm{mmHg}$. After obtaining the baseline FBF value, hyperaemic FBF was recorded after occluding the wrist with a pressure set at $250 \mathrm{mmHg}$ for $5 \mathrm{~min}$. FBF output signal was transmitted to a computer with Hokanson arterial inflow NIVP3 software, and the arterial blood flow signal was analysed at a later time by an independent reader blinded to the subject's history. By the method, FBF in the forearm is calculated as the volume of blood flow (in $\mathrm{ml}$ ) per $100 \mathrm{ml}$ of forearm tissue per minute. Forearm vasodilatory response to reactive hyperaemia (RH) was defined as the \% change in FBF from baseline to the maximum FBF during the period of $\mathrm{RH}$ as we have previously described in details ${ }^{(15)}$. It was previously shown that $\mathrm{RH}$ is endothelium dependent, and this method is widely used as an index of endothelial function in human forearm resistance vessels. It is considered to be a marker of NO bioavailability (since infusion of endothelial NO synthase inhibitor NG-monomethyl-L-arginine inhibits RH by approximately $50 \%)^{(15)}$.
Subjects rested in a supine position and were asked to remain in the same position $15 \mathrm{~min}$ before obtaining measurements for the examination of endothelium-independent dilation. Fifteen minutes after RH and while blood flow was confirmed to have returned to the baseline level, baseline measurements were obtained and subsequently $0.4 \mathrm{mg}$ of nitroglycerine (NTG) was sublingually administrated by a spray device. It is worth to mention that before measurements, all participants had a blood pressure test in order to avoid hypotensive response or other problems (subjects with systolic blood pressure lower than $105 \mathrm{mmHg}$ were excluded from NTG measurements). After baseline measurements, NTG was administered. A 2-3 min period of post-administration is adequate for NTG to act. Following this 2 min time, continuous measurements (curves) were obtained for the examination of endothelium-independent dilation. Forearm vasodilatory response to NTG was defined as the percentage change in FBF from baseline to the maximum FBF achieved after sublingual administration of NTG $0.4 \mathrm{mg}$, respectively ${ }^{(15)}$. NTG was expressed as the percentage change in FBF from baseline to the maximum FBF observed during the period post-NTG administration. Mean intra- and interobserver variabilities of RH measurements in our laboratory in thirty-seven healthy volunteers were $3 \cdot 1$ (SEM 1.7) and $4 \cdot 1$ (SEM 1.9$) \%$, respectively.

\section{Biochemical determinations}

Venous blood samples were taken at the beginning of each protocol and at $3 \mathrm{~h}$ post-intervention, before the final plethysmographic measurement, to avoid a possible effect of NTG administration on the measured parameters. After centrifugation at $1150 \mathrm{~g}$ at $4^{\circ} \mathrm{C}$ for $10 \mathrm{~min}$, serum was collected and stored at $-80^{\circ} \mathrm{C}$ until assayed. Total lipid PEROX were measured by using a commercially available photometric technique (kit by ImmunDiagnostik, Bensheim, Germany). The intra-assay $\mathrm{CV}$ was $3.1 \%$, while the inter-assay $\mathrm{CV}$ was $5.1 \%$ (a detection limit of $7 \mu \mathrm{mol} / \mathrm{l}$ ). Quantitative sandwich enzyme immunoassay technique was used to measure sVCAM-1 serum levels (ELISA kit by R\&D Systems, Wiesbaden, Germany). The intra-assay CV was $3.5 \%$, while the inter-assay CV $7.7 \%$ (a detection limit of $0.17-1.26 \mathrm{ng} / \mathrm{ml}$ ).

\section{Statistical analysis}

All variables were normally distributed, as confirmed by using the Kolmogorov-Smirnov test, and are expressed as means with their standard errors. Comparisons of baseline characteristics were performed by $\chi^{2}$ test for categorical variables or one-way ANOVA, followed by Bonferoni post hoc correction for multiple comparisons. The effect of treatment on each variable was examined by using ANOVA for repeated measures. Correlations between variables were performed by univariate analysis, and the Pearson's $r$ coefficient was estimated. A pre-study sample size calculation indicated that a sample of six patients was able to detect a $20 \%$ change in $\mathrm{RH}$ after an intervention, with a power of $90 \%$ and $\alpha=0 \cdot 05$. All analyses were carried out by using the SPSS v12.0 statistical package (SPSS Inc., Chicago, IL, USA).

The present study was conducted according to the guidelines laid down in the Declaration of Helsinki, and all 
procedures involving human subjects/patients were approved by the Institutional Ethics Committee. Written informed consent was obtained from all subjects/patients.

\section{Results}

The demographic characteristics and lipid profile of the participants are presented in Table 1 . There were no significant differences between the five groups at baseline.

\section{Effects on forearm blood flow measurements}

Acute consumption of maize oil, extra virgin olive oil, soya oil, cod liver oil or water had no significant effect on absolute FBF at rest or during post-ischaemic hyperaemia (Fig. 1). However, maize oil induced a significant decrease in endothelial function as that was evaluated by RH, Fig. 2) $1 \mathrm{~h}$ after consumption. An opposite effect was observed for cod liver oil and soya oil, which improved RH $1 \mathrm{~h}$ post-consumption (Fig. 2). Surprisingly, extra virgin olive oil had no effect on $\mathrm{RH}$ during the $3 \mathrm{~h}$ post-consumption (Fig. 2). The change in $\mathrm{RH}$ (from baseline to $1 \mathrm{~h}$ post-oil consumption) was significantly lower in maize oil-treated subjects compared to water-treated (control) subjects $(P<0 \cdot 05)$. The improvement of $\mathrm{RH}$ in the cod liver oil- and soya oil-treated subjects $1 \mathrm{~h}$ after consumption was significantly greater compared with controls $(P<0 \cdot 05)$. There was no significant difference in NTG between the all study groups $(P=\mathrm{NS})$.

\section{Effects on oxidative stress status and vascular inflammation}

There were no significant differences between PEROX values at baseline and $3 \mathrm{~h}$ after consumption in all study groups. PEROX remained unchanged in all groups during the $3 \mathrm{~h}$ period (Fig. 3(a)). Similarly, acute oil consumption had no effect on serum levels of sVCAM-1 (Fig. 3(b)), suggesting that the acute consumption of oils has no effect on vascular inflammation in healthy individuals.

\section{Discussion}

In the present study, we examined the effects of acute consumption of equivalent amount of extra virgin olive oil, soya oil, cod liver oil and maize oil on endothelial function adhesion molecules and oxidative stress status in a population of healthy adults. We found that acute consumption of maize oil leads to impaired endothelial function, while cod liver oil and soya oil may improve endothelial function in human subjects.

\section{Oil consumption and endothelial function}

Previous studies have shown that oil consumption may affect endothelial function. The results have been controversial and still not clear as the implicated mechanisms affecting endothelial function are still under investigation. Cortés et al. ${ }^{(16)}$ found that consumption of olive oil decreased flow-mediated dilatation in healthy subjects and hypercholesterolaemic patients after $4 \mathrm{~h}$. In controversy, flow-independent dilatation remained unchanged. Recent observations have shown that olive oils with different phenolic compounds content have different effects on endothelial function. Specifically, hypercholesterolaemic volunteers faced an improvement in ischaemic $\mathrm{RH}$ after high phenolic acid virgin olive oil ${ }^{(17)}$. Similar to Cortés et al., Rueda-Clausen et al. ${ }^{(18)}$ supported that both olive oil and soyabean oil reduced flow-mediated dilatation after $3 \mathrm{~h}$. Moreover, fish oil was superior to maize oil, according to its effects on endothelial function. Healthy volunteers fed with fish oil for a period of $14 \mathrm{~d}$ had a significant increase both in endothelial-dependent vasodilatation and endothelial-independent vasodilatation, while a similar effect did not exist with maize oil ${ }^{(19)}$. Despite the beneficial effects on healthy subjects, fish oil supplementation significantly changed endotheliumdependent coronary vasodilatation in heart transplant recipients, but not endothelium-independent vasodilatation ${ }^{(20)}$. Finally, cod liver oil (fish oil) appeared to delay the impairment of endothelium-dependent relaxations in hypercholesterolaemia and atherosclerosis models, partly due to improved release of endothelium-derived relaxing factor and improved relaxation of coronary smooth muscle to the factor ${ }^{(21)}$.

In the present study, we have shown that vascular endothelium responds in a different way to different types of oil, despite the equivalent amount of oil received. We observed that endothelium-dependent dilatation was significantly reduced $1 \mathrm{~h}$ after consumption of maize oil, while soya oil induced a significant improvement of endothelial function

Table 1. Demographic characteristics and lipid profile of the participants ${ }^{\star}$

(Mean values with their standard errors)

\begin{tabular}{|c|c|c|c|c|c|c|c|c|c|c|}
\hline & \multicolumn{2}{|c|}{ Maize oil } & \multicolumn{2}{|c|}{ Liver oil } & \multicolumn{2}{|c|}{ Olive oil } & \multicolumn{2}{|c|}{ Soya oil } & \multicolumn{2}{|c|}{ Water } \\
\hline & Mean & SEM & Mean & SEM & Mean & SEM & Mean & SEM & Mean & SEM \\
\hline Subjects (males/females) & $7(6 / 1)$ & & $8(5 / 3)$ & & $9(6 / 3)$ & & $8(6 / 2)$ & & $5(3 / 2)$ & \\
\hline Age (years) & $25 \cdot 8$ & $1 \cdot 0$ & $33 \cdot 1$ & $3 \cdot 6$ & $26 \cdot 0$ & 1.4 & 28.5 & $3 \cdot 1$ & 24.4 & 1.2 \\
\hline BMI $\left(\mathrm{kg} / \mathrm{m}^{2}\right)$ & 24.7 & $1 \cdot 1$ & 22.5 & $1 \cdot 2$ & 23.5 & 0.9 & $23 \cdot 3$ & $1 \cdot 2$ & $22 \cdot 7$ & $1 \cdot 2$ \\
\hline SBP (mmHg) & 114 & 2 & 105 & 2 & 108 & 2 & 107 & 3 & 113 & 3 \\
\hline $\mathrm{DBP}(\mathrm{mmHg})$ & 77 & 5 & 70 & 3 & 74 & 5 & 74 & 5 & 78 & 4 \\
\hline Cholesterol (mg/l) & 1526 & $80 \cdot 9$ & 1735 & 173 & 1780 & 166 & 1763 & 127 & 1924 & 103 \\
\hline TAG (mg/l) & 572 & 38 & 667 & $111 \cdot 2$ & 648 & 65 & 859 & 210 & 687 & $85 \cdot 7$ \\
\hline HDL (mg/l) & 493 & 30 & 548 & 69 & 471.4 & 46 & 494 & 44 & 594 & 35 \\
\hline LDL (mg/l) & 922 & 74 & 1056 & 114 & 1181 & 133 & 1097 & 96 & 1195 & 96 \\
\hline
\end{tabular}

SBP, systolic blood pressure; DBP, diastolic blood pressure.

* There was no significant difference in any of the examined baseline characteristics between the five groups. 
(b)
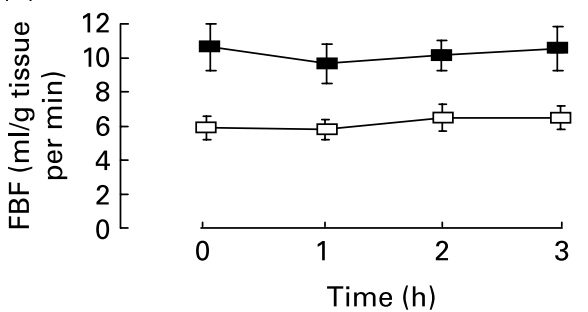

(d)

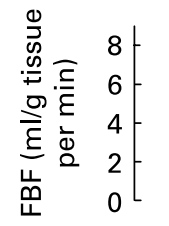

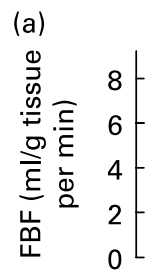

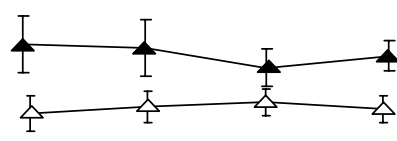

0

Time (h)

(c)

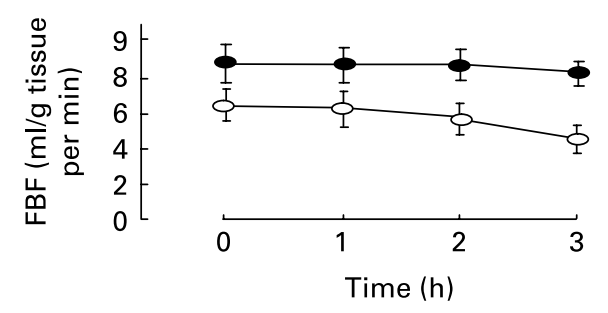

(e)

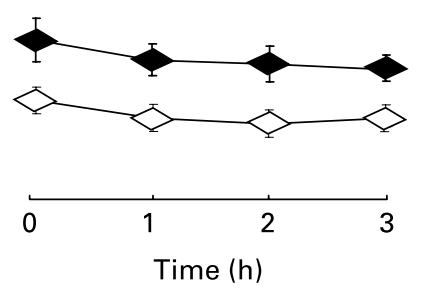

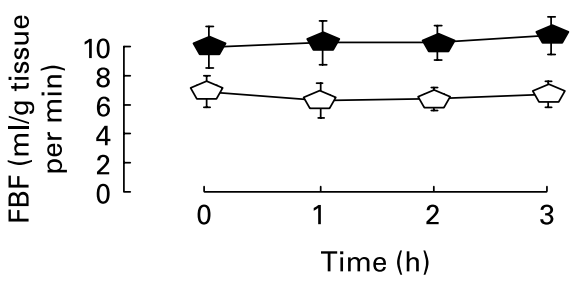

Fig. 1. There was no significant effect of (a) water ( $\boldsymbol{\Lambda}$, rest; $\Delta$, hyperaemia), (b) maize oil ( $\boldsymbol{\square}$, rest; $\square$, hyperaemia), (c) extra virgin olive oil ( $\bullet$, rest; $\bigcirc$, hyperaemia), (d) cod liver oil $(\longrightarrow$, rest; $\diamond$, hyperaemia) or (e) soya oil $(\square$, rest; $\square$, hyperaemia) on resting forearm blood flow (FBF) or maximum hyperaemic FBF during the first $3 \mathrm{~h}$ post-oil consumption.

$1 \mathrm{~h}$ after consumption. On the other hand, cod liver oil induced a borderline improvement of $\mathrm{RH} 1 \mathrm{~h}$ post-consumption. Interestingly, the present study showed that both the negative and positive effects of different oils consumption on endothelial function were diminished after $3 \mathrm{~h}$ time. This could be attributed to the fact that the healthy vascular endothelium, which was examined in the present study, has the potential to develop unknown defensive mechanisms after short time alterations (in 1-3h). Additionally, the fact that both inflammatory process and oxidative stress status were unaffected after $3 \mathrm{~h}$ could imply that the association between these processes and endothelial function was also unaffected. Finally, like other studies, the present study was designed so that baseline testing was performed in the early morning and post-prandial testing was done about $3 \mathrm{~h}$ later. Probably, the physiologic improvement of endothelial function during the morning counteracted any changes due to effect of the different oil consumption.

\section{Oil consumption, oxidative stress and inflammatory mechanisms}

Several studies have investigated the association between oil consumption and oxidative stress status. Cortés et al. ${ }^{(16)}$ were able to show such an association in hypercholesterolaemic patients and healthy individuals. Both the two groups faced a decrease in oxidative stress markers after olive oil consumption (as well as walnuts). It is well known that olive oil reduces oxidative stress due to polyphenols ${ }^{(7,22)}$. In addition, a single dose of olive oil resulted in an increase in plasma lipid peroxidation products reaching a peak at $4-6 \mathrm{~h}$ and returning to baseline values at $24 \mathrm{~h}$ after oil ingestion ${ }^{(23)}$. More recently, consumption of olive oil at real-life doses has improved the fatty acid profile in LDL, the changes being associated with a reduction in the oxidative damage to lipids ${ }^{(24)}$. In addition, diet modification with free virgin olive oil showed a significant reduction in oxidative stress markers after 3 months ${ }^{(14)}$.

Furthermore, Suzukawa et al. ${ }^{(25)}$ compared the effects of fish and maize oil on hypertensive patients. The results of the present study showed that from the standpoint of atherosclerosis, fish oil fatty acids adversely raise the susceptibility of LDL to $\mathrm{Cu}$-induced and macrophage-mediated oxidation. Maize oil had no effect on the points measured in the study. Other studies comparing the effects of different kind of oils reported that fish oil is much more beneficial than olive oil regarding its antioxidant properties. Interestingly, another study evaluated that soyabean oil had a higher antioxidant capacity than olive oil too ${ }^{(26)}$. Regarding to soya, a recent study has found that consumption of soya protein in conjunction with a high-fat meal does not modify acutely postprandial oxidative stress in young healthy men ${ }^{(27)}$.

Evidence suggests that dietary habits or modifications may affect inflammatory processes ${ }^{(28)}$. It has been shown that Mediterranean diet reduced the levels of inflammatory markers in patients with metabolic syndrome after a long-term period ${ }^{(29)}$. Similarly, $n-3$ fatty acids supplementation reduced sVCAM-1 

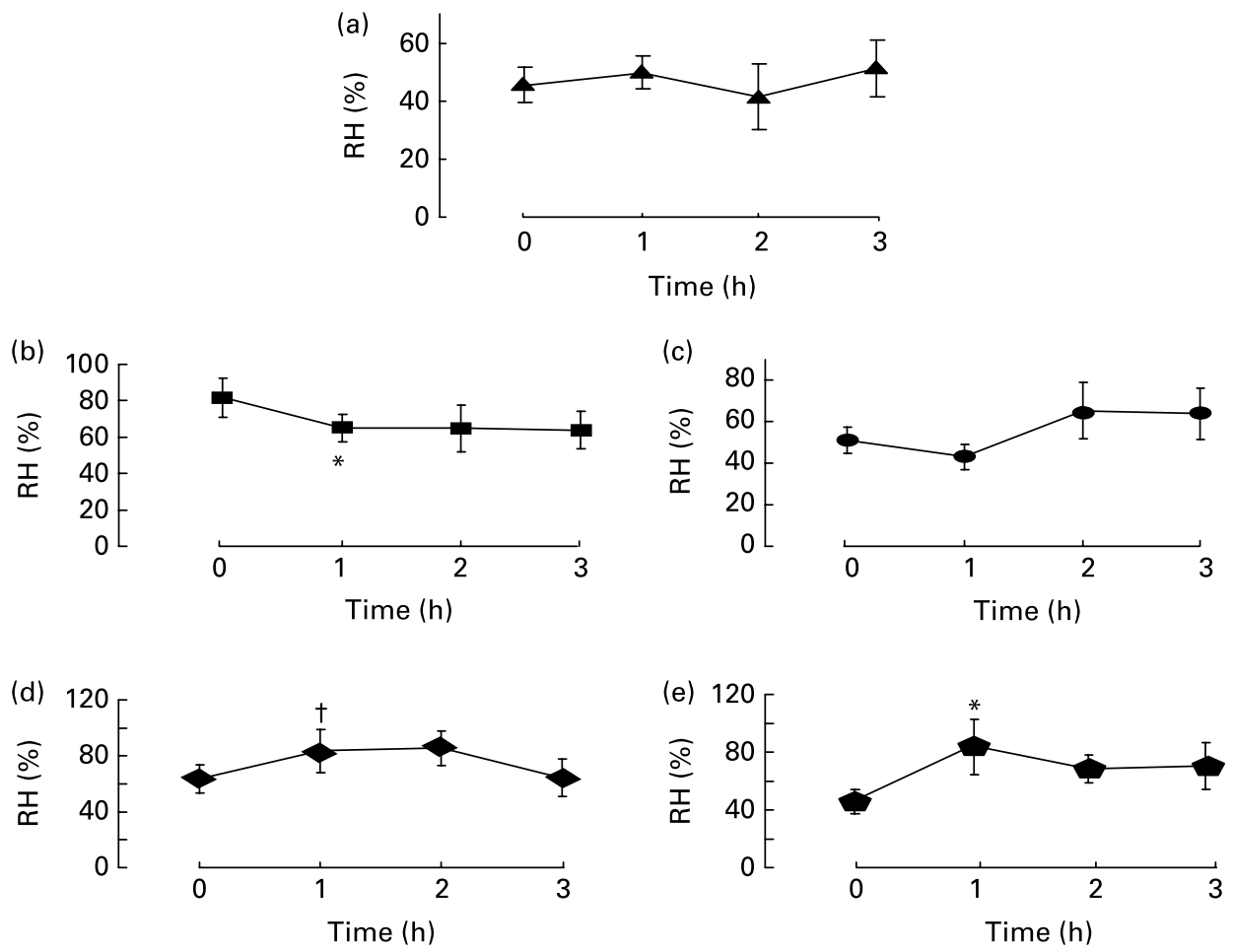

Fig. 2. (a) Forearm vasidilatory response to reactive hyperaemia $(\mathrm{RH})$ remained unchanged in the control group after water consumption ( $\mathbf{\Delta})$, while (b) it was sig-

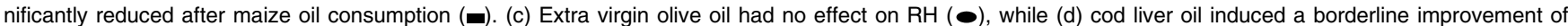

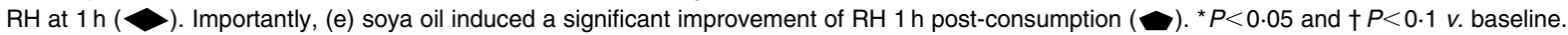

levels in patients with coronary artery disease ${ }^{(30)}$, which is a key adhesion molecule contributing to atherogenesis ${ }^{(10)}$ and a marker of endothelial injury and activation ${ }^{(31)}$.

In addition, Fuentes et al. ${ }^{(32)}$ showed that different types of fat composition meals affected differentially (in $4 \mathrm{~h}$ ) VCAM-1 levels, as MUFA appeared to be more beneficial than $\alpha$-linoleic acid by reducing VCAM-1 levels. However, data seem to be more confusing when referring to healthy adults, as fish oil had no impact on VCAM-1 levels after a 12-week period ${ }^{(33)}$. Furthermore, the effect of different types of diet (different fat composition) on inflammation has also been examined.
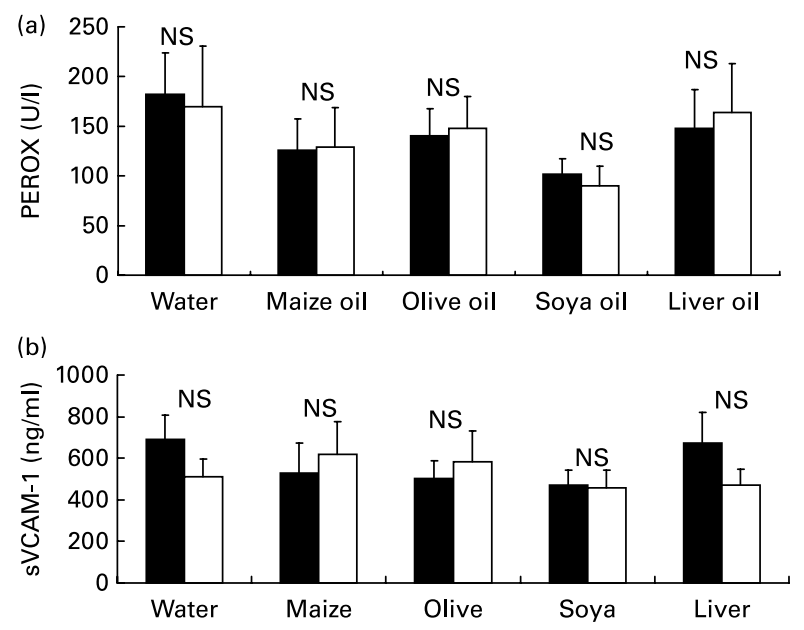

Fig. 3. (a) Total lipid peroxides (PEROX) as well as (b) soluble vascular cells adhesion molecule 1 (sVCAM-1) remained unchanged after oil consumptions in all study groups.
$\mathrm{NF}-\kappa \mathrm{B}$, which plays a key role in the inflammatory response, was diminished after Mediterranean diet. On the contrary, Western diet increased 2.7-fold NF-кB compared with the Mediterranean $\operatorname{diet}^{(34)}$. In addition, fish oil appeared to act beneficially in experimental model regarding to inflammation. Suppression of tumor necrosis factor- $\alpha$ and IL- 6 expression by fish oil was attributed to its inhibitory effect on NF- $\mathrm{B}$ activation, implicating that fish oil may ameliorate atherosclerosis by its immunomodulating effects ${ }^{(35)}$.

In the present study, we have found that different types of oil have no effect on oxidative stress status in healthy adults. Specifically, total lipid PEROX remained unchanged after consumption of maize oil, extra virgin olive oil, soya oil and liver oil. Similarly, none of the used types of oils affected serum levels of sVCAM-1, a finding that implies that despite the effect of maize oil, soya and cod liver oil on vascular endothelium, these types of oil have no impact on vascular inflammatory process. The decision for the measurement of VCAM-1 levels needs further justification. It is well documented that the expression of adhesion molecules such as sVCAM-1 is increased in the presence of impaired endothelial function, and the soluble form of this molecule (sVCAM-1) cleaved and released to the circulation is a marker of endothelial injury and activation ${ }^{(31)}$. Therefore, by examining the impact of oils consumption on endothelial function (assessed by plethysmography), we evaluated NO release by vascular endothelium; while by measuring the changes in sVCAM-1, we searched for a possible effect on endothelial cell pro-atherogenic activation.

On the other hand, serum levels of sVCAM-1 are rapidly affected by various interventions, mainly due to the rapid 
cleavage of the soluble form of this molecule from endothelial surface and its release to the circulation, after endothelial activation. A rapid response of circulating sVCAM-1 after endothelial activation was documented by various studies in the past, and it seems that a period of $3-4 \mathrm{~h}$ is enough to observe this effect after an acute intervention ${ }^{(32)}$.

In conclusion, we have demonstrated that acute maize oil consumption blunts endothelial function in healthy individuals. On the contrary, acute consumption of codfish oil or soya oil slightly improves endothelial function $1 \mathrm{~h}$ after consumption, while olive oil has a rather neutral effect. The neutral effects of olive oil should be considered with caution as major components such as oleic acid act beneficially on endothelial function, while preventing from further alterations in inflammatory process and oxidative stress. In addition, other minor components of extra virgin olive oil such as oleuropein, hydroxytyrosol and tyrosol are strong antioxidants and radical scavengers, thus appearing to be protective for the vascular endothelium. However, such an effect was not observed in the present study. Most importantly, we observed that none of the four types of oil had an impact on PEROX and vascular adhesion molecule levels, suggesting that the observed effect on endothelial function is largely independent of oxidative stress alterations and vascular inflammatory process in healthy, young and low-risk individuals.

\section{Study limitations}

The present study showed that consumption of maize oil leads to impaired endothelial function, while soya oil and cod liver oil slightly improve endothelial function. However, no type of oils affected inflammatory process and systemic oxidative stress, suggesting that their effect on endothelial function may not be mediated by free radicals' bioavailability. Despite the fact that the results of the present study are indicative of the effects, that different types of oil exert on endothelial function, our findings cannot be widely generalised as there are some points that need further investigation and evaluation. Thus, the present study is characterised by a short follow-up period, as it investigates the acute effects of different types of oil. A longer follow-up period may have given different results. In addition, the study population was relatively small. However, the present study was adequately powered to identify significant differences. A pre-study sample size calculation indicated that a sample of six patients was able to detect a $20 \%$ change in RH after an intervention, with a power of $90 \%$ and $\alpha=0 \cdot 05$. Finally, another limitation of the present study could be the absence of data regarding the lipid profile changes in the participants after oil consumption. Although this is an important issue to address, previous studies showed controversial results and this was the reason for not measuring post-prandial lipid levels.

\section{Acknowledgements}

The present research received no specific grant from any funding agency in the public, commercial or not-for-profit sectors.

D. T. designed the study and wrote the manuscript. N. P. wrote the manuscript and collated all statistical information. C. A. designed the study and wrote the manuscript. A. G., G. B., P. G., E. S., A. M., G. S., T. P. and C. T. collated all statistical information. C. S. designed the study. All authors read and approved the findings of the study. Disclosures. There is no disclosure or any potential conflict of interest.

\section{References}

1. Libby P \& Theroux P (2005) Pathophysiology of coronary artery disease. Circulation 111, 3481-3488.

2. Tousoulis D, Antoniades C, Tentolouris C, et al. (2002) L-Arginine in cardiovascular disease: dream or reality? Vascular Med 7, 203-211.

3. Davignon J \& Ganz P (2004) Role of endothelial dysfunction in atherosclerosis. Circulation 109, Suppl III, III-27-III-32.

4. Celermajer DS, Sorensen KE, Bull C, et al. (1994) Endothelium-dependent dilation in the systemic arteries of asymptomatic subjects relates to coronary risk factors and their interactions. J Am Coll Cardiol 24, 1468-1474.

5. Ludmer PL, Selwyn AP, Shook TL, et al. (1986) Paradoxical vasoconstriction induced by acetylcholine in atherosclerotic coronary arteries. $N$ Engl J Med 315, 1046-1051.

6. Reddy KG, Nair RN, Sheehan HM, et al. (1994) Evidence that selective endothelial dysfunction may occur in the absence of angiographic or ultrasound atherosclerosis in patients with risk factors for atherosclerosis. J Am Coll Cardiol 23, 833-843.

7. Droge W (2002) Free radicals in the physiological control of cell function. Physiol Rev 82, 47-95.

8. Hsich E, Segal BH, Pagano PJ, et al. (2000) Vascular effects following homologous disruption of $447^{\text {PHOX: an essential }}$ component of NADPH oxidase. Circulation 101, 1234-1236.

9. Ogita H \& Liao J (2004) Endothelial function and oxidative stress. Endothelium 11, 123-132.

10. Tousoulis D, Antoniades C \& Stefanadis C (2007) Assessing inflammatory status in cardiovascular disease. Heart 93, $1001-1007$.

11. Carluccio MA, Massaro M, Scoditti E, et al. (2007) Vasculoprotective potential of olive oil components. Mol Nutr Food Res 51, $1225-1234$.

12. Vogel R, Coretti M \& Plotnick G (2000) The postprandial effect of components of the Mediterranean diet on endothelial function. J Am Coll Cardiol 36, 1455-1460.

13. Covas MI (2008) Bioactive effects of olive oil phenolic compounds in humans: reduction of heart disease factors and oxidative damage. Inflammopharmacology 16, 216-218.

14. Fitó M, Guxens M, Corella D, et al. (2007) Effect of a traditional Mediterranean diet on lipoprotein oxidation: a randomized controlled trial. Arch Intern Med 167, 1195-1203.

15. Tousoulis D, Antoniades C \& Stefanadis C (2005) Evaluating endothelial function in humans: a guide to invasive and non-invasive techniques. Heart 91, 553-558.

16. Cortés B, Núñez I, Cofán M, et al. (2006) Acute effects of high-fat meals enriched with walnuts or olive oil on postprandial endothelial function. J Am Coll Cardiol 48, 1666-1671.

17. Ruano J, Lopez-Miranda J, Fuentes F, et al. (2005) Phenolic content of virgin olive oil improves ischemic reactive hyperemia in hypercholesterolemic patients. $J$ Am Coll Cardiol 46, 1864-1868.

18. Rueda-Clausen CF, Silva FA, Lindarte MA, et al. (2007) Olive, soybean and palm oils intake have a similar acute detrimental effect over the endothelial function in healthy young subjects. Nutr Metab Cardiovasc Dis 17, 50-57.

19. Shah AP, Ichiuji AM, Han JK, et al. (2007) Cardiovascular and endothelial effects of fish oil supplementation in healthy volunteers. Cardiovasc Pharmacol Ther 12, 213-219.

20. Fleischhauer FJ, Yan WD \& Fischell TA (1993) Fish oil improves endothelium-dependent coronary vasodilation in heart transplant recipients. J Am Coll Cardiol 21, 982-989. 
21. Shimokawa H \& Vanhoutte PM (1998) Dietary cod-liver oil improves endothelium-dependent responses in hypercholesterolemic and atherosclerotic porcine coronary arteries. Circulation 78, $1421-1430$.

22. Covas MI, Nyyssönen K, Poulsen HE, et al. (2006) The effect of polyphenols in olive oil on heart disease risk factors: a randomized trial. Ann Intern Med 145, 333-341.

23. Fitó M, Gimeno E, Covas MI, et al. (2002) Postprandial and short-term effects of dietary virgin olive oil on oxidant/antioxidant status. Lipids 37, 245-251.

24. Cicero AF, Nascetti S, López-Sabater MC, et al. (2008) Changes in LDL fatty acid composition as a response to olive oil treatment are inversely related to lipid oxidative damage: The EUROLIVE study. J Am Coll Nutr 27, 314-320.

25. Suzukawa M, Abbey M, Howe PR, et al. (1995) Effects of fish oil fatty acids on low density lipoprotein size, oxidizability, and uptake by macrophages. J Lipid Res 36, 473-484.

26. Pellegrini N, Serafini M, Colombi B, et al. (2003) Total antioxidant capacity of plant foods, beverages and oils consumed in Italy assessed by three different in vitro assays. $J$ Nutr 133, $2812-2819$

27. Campbell CG, Brown BD, Dufner D, et al. (2006) Effects of soy or milk protein during a high-fat feeding challenge on oxidative stress, inflammation, and lipids in healthy men. Lipids $\mathbf{4 1}$, 257-265.

28. Estruch R, Martínez-González MA, Corella D, et al. (2006) Effects of a Mediterranean-style diet on cardiovascular risk factors: a randomized trial. Ann Intern Med 145, 1-11.
29. Esposito K, Marfella R, Ciotola M, et al. (2004) Effect of a Mediterranean-style diet on endothelial dysfunction and markers of vascular inflammation in the metabolic syndrome: a randomized trial. JAMA 292, 1440-1446.

30. Johansen O, Seljeflot I, Høstmark A, et al. (1999) The effect of supplementation with omega-3 fatty acids on soluble markers of endothelial function in patients with coronary heart disease. Arterioscler Thromb Vasc Biol 19, 1681-1686.

31. O'Brien KD, McDonald TO, Chait A, et al. (1996) Neovascular expression of E-selectin, intercellular adhesion molecule-1 in human atherosclerosis and their relation to intimal leukocyte content. Circulation 93, 672-682.

32. Fuentes F, López-Miranda J, Pérez-Martínez P, et al. (2008) Chronic effects of a high-fat diet enriched with virgin olive oil and a low-fat diet enriched with a-linolenic acid on postprandial endothelial function in healthy men. Br J Nutr 100, 159-165.

33. Miles EA, Thies F, Wallace FA, et al. (2001) Influence of age and dietary fish oil on plasma soluble adhesion molecule concentrations. Clin Sci (Lond) 100, 91-100.

34. Perez-Martinez P, Lopez-Miranda J, Blanco-Colio L, et al. (2007) The chronic intake of a Mediterranean diet enriched in virgin olive oil, decreases nuclear transcription factor kappaB activation in peripheral blood mononuclear cells from healthy men. Atherosclerosis 194, 141-146.

35. Ma H, Wang J, Wang J, et al. (2007) Fish oil ameliorates the allograft arteriosclerosis of intestine on rats. Pediatr Transplant 11, 173-179. 